\title{
Two Cases of Pneumatic Retinopexy as a Primary Successful Intervention of Rhegmatogenous Retinal Detachment
}

\author{
Enkhtuul Sedbazar, Anarsaikhan Narmandakh, Gan-erdene Mandakhbayar, Byambadorj Dulamjav
}

Department of Ophthalmology, First Central Hospital of Mongolia, Ulaanbaatar, Mongolia

Purpose: We present a case series of macula involving or non-involving rhegmatogenous retinal detachment successfully managed with pneumatic retinopexy with additional cryopexy and laserpexy as a primary single operation.

Case summary: A review of two patients with visually significant macula off rhegmatogenous retinal detachment which involves more than 4 clock hours in expansion was conducted. At presentation, all two were underwent a pneumatic retinopexy with expansile Perfluoropropane pure gas $0.5 \mathrm{~mL}$ bubble with proper cryopexy and maintenance of appropriate head position regimen without intraoperative complications. The following day, laser was applied around the margin of the retinal break and intraocular pressure was monitored closely. During postoperative evaluation, the eyes achieved a good visual acuity and fundus examination revealed a fully resolved subretinal fluid and reattached retina. During short term follow-up, the retina remained completely attached and no further complications were detected.

Conclusions: There is no significant drawback in trying pneumatic retinopexy primary in eyes that encounter the selection criteria. If a pneumatic retinopexy fails, a prompt following operation should be performed, the final anatomical and visual outcomes are not disadvantaged by the initial pneumatic procedure.

Keywords: Cryoretinopexy; Perfluoropropane gas; Pneumatic retinopexy; Retinopexy; Rhegmatogenous retinal detachment

\section{Introduction}

Pneumatic Retinopexy is a technically easy, minimally invasive and cost-effective procedure for the treatment of the indicated cases of rhegmatogenous retinal detachment (RRD) when compared with other surgical approaches [1,2]. This procedure is also associated with reduced morbidity and faster postoperative recovery and a more natural reattachment of the retina compared to pars plana vitrectomy and scleral buckling. Proper patient selection depending upon the size, number, location, type and extension of the retinal breaks are critical to the success rate of procedure [3,4]. This intervention remains a useful procedure for treating certain types of primary RRDs. Anatomic success rates of $90 \%$ can be achieved when strict selection criteria are applied [5]. Superior visual acuity outcomes have also been reported [4]. Intraoperative complications are primarily related to the intraocular pressure increase caused by gas injection, or the misdirection of the gas. There is a low rate $(0-4 \%)$ of postoperative complications involving improper location of the injected air or gas, generally into the subretinal space [6]. In Mongolia, we commenced to perform a pneumatic retinopexy procedure since 2019 . Since this time

\section{Address reprint requests to Enkhtuul Sedbazar, MD}

Department of Ophthalmology, First Central Hospital of Mongolia, S. Zorig Street-2, Sukhbaatar District, Ulaanbaatar 210648, Mongolia

Tel: 976-98-885-688

E-mail: s.enkhtuul@fchm.edu.mn
Received: 2021. 2. 24.

Revised: 2021. 6. 20.

Accepted: 2021. 7. 12. 
period, a total of 14 patients underwent pneumatic retinopexy, which in two cases out of them were documented with images. It offers a cost-effective and useful technique for repairing some indicated cases of primary rhegmatogenous retinal detachment especially in low-resource countries. Generally patients are more likely to present late and complex detachments are relatively common in Mongolia. Despite this issues pneumatic retinopexy results are encouraging with high final anatomical success and reattached retinas. Herein, we conducted patients who had a 5-6-month of postoperative follow-ups to determine factors predictive of short-term functional outcome and anatomic success after pneumatic retinopexy repair.

\section{Case Report}

\section{Case 1}

A 63-year-old Mongolian woman presented complaining of the painless diminution of vision in the left eye sudden in onset, progressed for 20 days and then was worsen stationary for the last 10 days. She underwent cataract surgeries in both eyes. Preoperative eye evaluation summarized in Table 1 and Fig. 1.

The clinical diagnosis was made as following Macula off rhegmatogenous retinal detachment of left eye, pseudophakia of the both eyes.

First, the patient underwent a thorough scleral-depressed peripheral retinal examination to identify all retinal pathologic features. The procedure was performed under local (subconjunctival) anesthesia and aseptic technique. An anterior

Table 1. Preoperative eye exam

\begin{tabular}{lcc}
\hline & Right eye & Left eye \\
\hline BCVA & $6 / 6$ & HM \\
IOP $(\mathrm{mmHg})$ & 15 & 7 \\
AL $(\mathrm{mm})$ & 23.58 & 18.42 \\
RAPD & Nil & Positive \\
DFE & Within normal & Shaffer's sign / $/$, Weiss ring $/+$ /, \\
& limits & Macula involving \\
& & (Mac-off) superior quadrant (10-4 \\
& & o'clock) RRD with single horse- \\
& & shoe tear at 2 clock
\end{tabular}

$\mathrm{BCVA}=$ best corrected visual acuity; HM = hand motion; IOP = intraocular pressure; $\mathrm{AL}=$ axial length; $\mathrm{RAPD}=$ relative afferent pupillary defect; DFE = dilated fundus examination; RRD = rhegmatogenous retinal detachment. chamber paracentesis was performed $(0.3 \mathrm{~mL}$ anterior chamber [AC] fluid was evacuated) using a 30-gauge needle on a plungerless $1 \mathrm{~mL}$ syringe followed by $0.5 \mathrm{~mL}$ of pure $100 \%$ Perfluoropropane gas $\left(\mathrm{C}_{3} \mathrm{~F}_{8}\right)$ was injected through the pars plana (3.5-4 mm from limbus), into the lower nasal region (at 7 o'clock) away from the retinal break and the detached area using a 30-gauge needle on $1 \mathrm{~mL}$ syringe then kept the patient face down positioning immediately. Five minutes after this stage, the intraocular pressure and fundus were evaluated. Intraocular pressure and retinal vessels pulsation on the optic nerve head were tolerable. Following this, the patient was instructed to position her face down for 6 hours followed by her head and chin were inclined upwards slightly over the right shoulder in the sitting or right tilted head up position so that the bubble is opposed to the break for 12 to 24 hours. Then the patient was told to maintain the position as much as possible for another 2 to 3 days. Because of bullous detachment of the retina, cryopexy was not able to be done at the same time of gas injection. The day after retina was less bullous and cryoretinopexy was applied once surrounding to the edge of retinal break at the position of 2 o'clock for sealing the retina. Considering the chorioretinal strengthening and scarring time after applying the cryo, a day after laser photocoagulation (200 $\mu \mathrm{m}$ spot sizes, $160 \mathrm{mV}$ power) was applied around the tear and 360 degrees peripheral retina.

During follow-up at 7 th day postoperative pneumatic retinopexy, there was an improvement in visual acuity of 6/15 in the left eye. On fundoscopy, the retina was reattached, subretinal fluid (SRF) was significantly resolved and no unidentified breaks detected (Fig. 2). The patient was seen at 1,3 , and 6 months postoperatively and remained stable. At a 6-month follow-up, the visual acuity enhanced to 6/8.5 with the attached retina and normal intraocular pressure (IOP) of

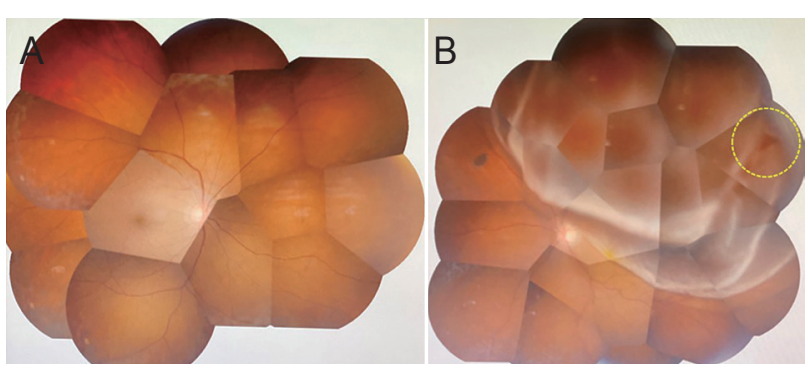

Figure 1. Montage color fundus photograph of the right eye showed normal (A), and the left eye demonstrated macula off RRD, which spans 6 clock hours with a tear in superotemporal quadrant (yellow circle) (B). $\mathrm{RRD}=$ rhegmatogenous retinal detachment. 
$13 \mathrm{mmHg}$ (Table 2).

\section{Case 2}

A 50-year-old Mongolian woman presented with chief complaint of sudden appearance of a dark curtain over a temporal portion of the visual field in the left eye. She reported excellent vision in left eye until 5 days prior, when she first noticed floaters suddenly in the left eye. The eyes exam explained at Table 3 and Fig. 3. The diagnosis was made as following Macula off rhegmatogenous retinal detachment of left phakic eye, mild myopia of the both eyes.

During the retinal peripheral examination, superior lattice

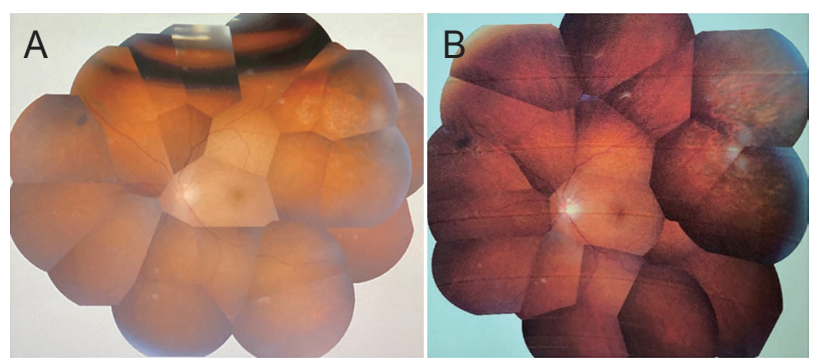

Figure 2. Montage color fundus photograph illustrates reattached retina with residual gas bubble after PR at 1 month (A). Completely absorbed gas and chorioretinal adhesion at 6-month follow-up (B). PR = pneumatic retinopexy.

Table 2. Postoperative visual acuity and IOP

\begin{tabular}{lccccc}
\hline & Day 1 & Day 7 & Month 1 & Month 3 & Month 6 \\
\hline LE BCVA & $6 / 50$ & $6 / 20$ & $6 / 15$ & $6 / 8.5$ & $6 / 8.5$ \\
IOP $(\mathrm{mmHg})$ & 18 & 16 & 18 & 14 & 13 \\
\hline
\end{tabular}

$\mathrm{IOP}=$ intraocular pressure; $\mathrm{LE} B C V A=$ best corrected visual acuity of the left eye. degeneration with hole in the right eye and inferior lattice degeneration in the attached retina in the left eye were found. So, we treated first lattice degeneration with holes in both eyes by laser. Laser retinopexy was applied in both eyes for right superior lattice degeneration with hole and left inferior lattice degeneration in the attached retina before pneumatic retinopexy. Retinal detachment was shallow, that is why then the break in the detached retina was treated with cryotherapy before gas injection. Third, gas injection was performed according to the technique previously described for the first case and the patient was instructed to maintain the head position. Finally, laser retinopexy was applied to the exciting tear on the following days. The patient was monitored at 1, 3, and 6 months postoperatively and the retina was completely reattached (Fig. 4). At 5-month follow up, visual acuity improved to $6 / 10$ and intraocular pressure was normalized to $12 \mathrm{mmHg}$ (Table 4). During these follow ups, the patient has not developed cataract progression in the left eye.

\section{Discussion}

Geographically, pneumatic retinopexy is not commonly performed in Asia, Europe and United Kingdom region with the majority of physicians perform the procedure less than once a month $[7,8]$. Several studies have suggested the growing trend toward vitrectomy as the preferred primary surgical intervention for primary RRD, vitrectomy was over twice as popular in cases of pseudophakic, macula-off RRD with posterior capsular opacity $[9,10]$. In USA especially the Northeast region demonstrated a greater preference for pneumatic retinopexy when compared to all other regions,

Table 3. Preoperative eye exam

\begin{tabular}{lcl}
\hline & Right eye & \multicolumn{1}{c}{ Left eye } \\
\hline BCVA & $6 / 6.6$ & HM \\
RAPD & Nil & Positive \\
IOP $(\mathrm{mmHg})$ & 8 & 23.08 \\
$\mathrm{AL}(\mathrm{mm})$ & 24.22 & Orrhage, macula off superior RRD \\
DFE & Lattice degeneration with hole at & Pigment floating in the anterior vitreous, complete PVD $/+$ /, mild vitreous hem- \\
& $11-12$ o'clock & Single horseshoe tear at 1:30 o'clock \\
& & Lattice degeneration at 6 o'clock in the attached retina
\end{tabular}

$\mathrm{BCVA}=$ best corrected visual acuity; $\mathrm{HM}=$ hand motion; $\mathrm{RAPD}=$ relative afferent pupillary defect; $\mathrm{IOP}=$ intraocular pressure; $\mathrm{AL}=$ axial length; $\mathrm{DFE}=$ dilated fundus examination; $\mathrm{PVD}=$ posterior vitreous detachment; $\mathrm{RRD}=$ rhegmatogenous retinal detachment. 
also revealed a trend toward a lower preference for pars plana vitrectomy compared to the West and South because of

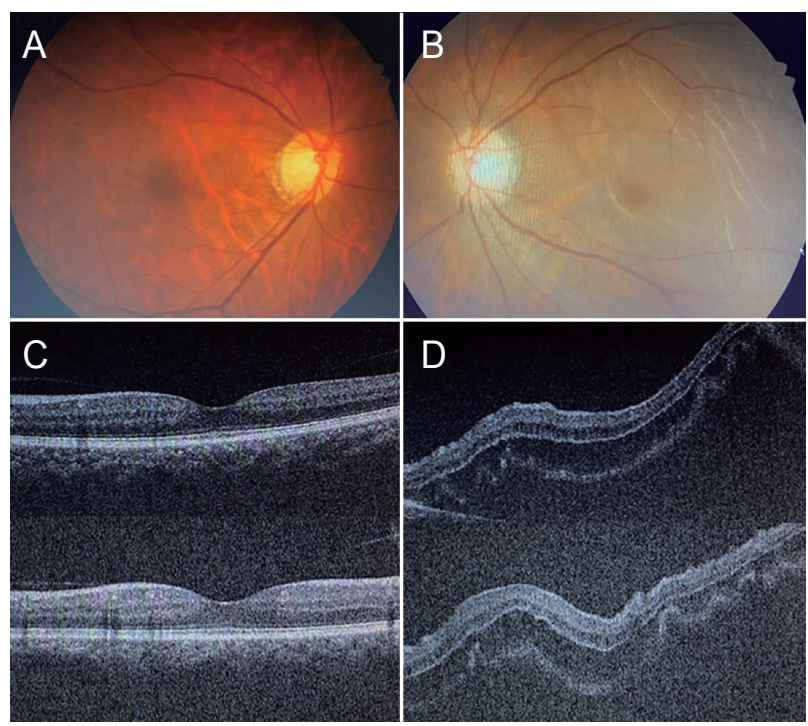

Figure 3. Color fundus photograph of normal right eye (A) and macula-off RRD with corrugated and undulated retinal folds in superior quadrant of the left eye (B). Optical coherence tomography imaging shows normal right eye (C). OCT illustrates an evidence of outer retinal folds, separated photoreceptors from RPE layer and subretinal fluid in the left eye (D). RRD = rhegmatogenous retinal detachment; OCT = optical coherence tomography; RPE = retinal pigment epithelium.
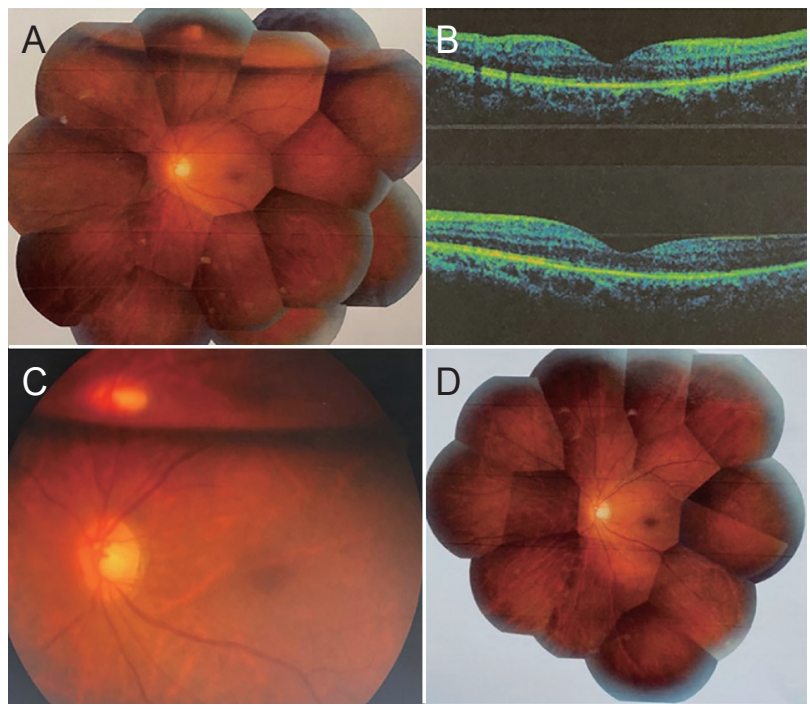

Figure 4. Color fundus photograph indicates reattached retina with residual gas bubble after PR at 7 and 20 days in left eye (A, C). The retina remains attached with complete resolution of outer retinal folds were revealed by OCT after procedure (B). Reattached retina of the left eye and no new retinal breaks detected at 5-month follow-up (D). PR = pneumatic retinopexy; $\mathrm{OCT}$ = optical coherence tomography. operating room burden and physician treatment preference.

Regarding pneumatic retinopexy, studies have indicated a single operation success rate of approximately 80 percent and greater than 95 percent with reoperations [11,12]. Ideal case selection and peripheral 360 degrees retinopexy can increase the single operation success rate to $97 \%$. The lower success rates associated with the latter two conditions are thought to be related to the presence of multiple small missed or new peripheral retinal breaks, frequently in conjunction with peripheral vitreoretinal traction $[13,14]$.

Ellakwa [15] reported their long-term results for 40 cases of retinal detachment treated with pneumatic retinopexy. Forty eyes of 40 patients with primary RRD due to a single U-shaped break in 28 eyes $(70 \%)$ or round hole/s in 12 eyes (30\%) occupying less than 1 clock-hour of the upper quadrant of the retina were included in the study retrospectively after treatment by pneumatic retinopexy. Mean postoperative best corrected visual acuity (BCVA) in patients with successful reattachment by pneumatic retinopexy was 0.40 \pm 0.21 after 3 years, while the mean postoperative BCVA in patients who required another procedure was $0.22 \pm 0.13$ at the 3-year follow-up [15].

Davis et al. [5] reported the result of pneumatic retinopexy procedure involving two hundred thirteen patients (mean follow-up 24.6 months) demonstrated that $64.8 \%$ of patients had a successful pneumatic retinopexy and vitreous hemorrhage and retinal detachment greater than 4.5 clock hours were the two factors that significantly affected successful outcome ( $p$ $=0.04$ and 0.01 , respectively). The overall mean final visual acuity was 20/40, with a mean of 20/30 in the success group and a mean of 20/60 in the failure group ( $p=0.001)$ [5].

Pneumatic retinopexy should be considered the first line treatment for RRD in patients fulfilling pneumatic retinopexy versus vitrectomy for the management of primary rhegmatogenous retinal detachment outcomes randomized trial recruitment criteria. Primary anatomic success at 12 months was achieved by $80.8 \%$ of patients undergoing pneumatic

Table 4. Postoperative visual acuity and IOP

\begin{tabular}{lccccc}
\hline & Day 1 & Day 7 & Month 1 & Month 3 & Month 5 \\
\hline LE BCVA & $6 / 60$ & $6 / 60$ & $6 / 40$ & $6 / 30$ & $6 / 10$ \\
IOP $(\mathrm{mmHg})$ & 14 & 13 & 15 & 12 & 12 \\
\hline
\end{tabular}

$\mathrm{IOP}=$ intraocular pressure; LE BCVA = best corrected visual acuity of the left eye. 
retinopexy versus $93.2 \%$ undergoing pars plana vitrectomy, with $98.7 \%$ and $98.6 \%$, respectively, achieving secondary anatomic success. Pneumatic retinopexy offers superior visual acuity, less vertical metamorphopsia, and reduced morbidity when compared with pars plana vitrectomy [4].

Martínez-Mujica et al. [16] identified three systematic reviews including six studies overall, of which three were randomized trials and concluded the anatomic result might be better with scleral buckling in terms of retinal reattachment and risk of recurrence, but the risk of ocular adverse events might be lower with pneumatic retinopexy.

Tanaka and Goldberg [17] described a case which relieving traction without retinopexy or pneumatic alone may be is sufficient for retinal detachment repair in selected cases. Fifty-seven-year-old woman (visual acuity [VA] of right eye 6/9.5) had fovea-sparing retinal detachment with a single horseshoe break at 11 o'clock and fluid tracking into the macula. $0.4 \mathrm{~mL} \mathrm{SF}_{6}$ was injected without retinopexy. On postoperative day 7 , right eye had a visual acuity of $6 / 6$ and the retina was grossly flat. Superior temporal retinal tear had operculated and traction was released [17]. Suzuki et al. [18] reported a case of successful pneumatic retinopexy for retinal detachment associated with a progressive superior temporal retinoschisis.

Giansanti et al. [19] reported a case of successful pneumatic retinopexy in an eye with large choroidal coloboma.

In our cases proper cryopexy and laser photocoagulation that allow chorioretinal adhesion serve as a barrier to additional fluid entering the subretinal space were the core principle of our successful retinal detachment repair and anatomical reattachment of the retina with a single intervention.

Several previous reports indicated decreased success rate in patients with pseudophakic and/or aphakic eyes when compared with patients with phakic eyes. In the group who need additional operations, pseudophakic eyes demonstrated a higher preoperative risk of failure. Phakic eyes fared better than aphakic eyes, with the single-operation successes of 71$84 \%$ for the former and $41-67 \%$ for the latter [13].

This case series aimed to present the short-term structural and functional outcome of pneumatic retinopexy in RRD in two patients after an average follow-up period of 5-6 months. In our cases involve proper cryotherapy and laser photocoagulation, in addition to endotamponade gas of $\mathrm{C}_{3} \mathrm{~F}_{8}$ with the maintenance of an appropriate head position. Generally, patients are more likely to present late, and complex detach- ments are relatively common in Mongolia. Despite these issues, the pneumatic retinopexy results are encouraging with higher final anatomical success, and re-attached retinas obtaining vision of $6 / 10$ or better. We already know that several previous reports indicated decreased success rate in patients with pseudophakic and/or aphakic eyes when compared with patients with phakic eyes.

Pseudophakic patients tend to have small tiny breaks near ora serrata which mean they can open up and you can miss breaks. So, it is essential to do scleral-depressed peripheral retinal examination to identify all retinal pathologic features carefully before the procedure. Pneumatic retinopexy could still be done in these pseudophakic patients. In the most patients pneumatic retinopexy will either work or at least decrease the SRF which makes the next surgery much easier. If it works then perfect, if not then you can do surgery and often it is much easier because they are less bullous.

Pneumatic retinopexy could be an alternative in eyes that encounter the selection criteria. If a pneumatic retinopexy fails, a following operation is needed timely. The final anatomical and visual outcomes are not disadvantaged by the initial pneumatic retinopexy procedure. The findings of these case study suggest that careful case selection, proper postoperative positioning and additional cryopexy and laser retinopexy has been considered as a primary treatment in selected cases of RRD.

\section{Informed Consent}

Written informed consent was obtained from the patient for publication of this case report and any accompanying images. An Approval of Research Ethical Control Board of Mongolian National University of Medical Science was obtained (REB No. 2020/3-08).

\section{Acknowledgments}

The authors are grateful to Dr. Sun Taek Lim for orienting and encouraging us to submit this case series to Journal of Retina. Also, we would like to express another special thanks to Dr. Sun Teak Lim and his team for training Mongolian Ophthalmologists at LEE YEON Eye Hospital, Gwangju, South Korea in 2009 which was the initial training in the field of Vitreo-Retina. This vitreo-retinal training in South Korea was a cornerstone to establishing and devel- 
oping the healthcare for patients suffering from a variety of vitreo-retinal diseases in Mongolia.

\section{Conflict of Interest}

The authors declare no conflict of interest relevant to the content of this article.

\section{References}

1. Jung JJ, Cheng J, Pan JY, et al. Anatomic, visual, and financial outcomes for traditional and nontraditional primary pneumatic retinopexy for retinal detachment. Am J Ophthalmol 2019;200:187200.

2. Stewart S, Chan W. Pneumatic retinopexy: patient selection and specific factors. Clin Ophthalmol 2018;12:493-502.

3. Holz ER, Mieler WF. Pneumatic retinopexy for primary retinal detachment. In: Kreissig I, ed. Primary Retinal Detachment, Options for Repair. Berlin: Springer-Verlag, 2005; chap. 4.

4. Hillier RJ, Felfeli T, Berger AR, et al. The pneumatic retinopexy versus vitrectomy for the management of primary rhegmatogenous retinal detachment outcomes randomized trial (PIVOT). Ophthalmology 2019;126:531-9.

5. Davis MJ, Mudvari SS, Shott S, Rezaei KA. Clinical characteristics affecting the outcome of pneumatic retinopexy. Arch Ophthalmol 2011;129:163-6.

6. Abecia E, Pinilla I, Olivan JM, et al. Anatomic results and complications in a long-term follow-up of pneumatic retinopexy cases. Retina 2000;20:156-61.

7. Assi AC, Chateris DG, Gregor ZJ. Practice patterns of pneumatic retinopexy in the United Kingdom. Br J Ophthalmol 2001;85:244.

8. Michelle Dalton E. Global trends in retina: how surgeons are practicing in 2018 [Internet]. Modern Retina; 2018 [cited 2018 Jul 24]. Available from: https://www.modernretina.com/view/glob- al-trends-retina-how-surgeons-are-practicing-2018.

9. Cho GE, Kim SW, Kang SW; Korean Retina Society. Changing trends in surgery for retinal detachment in Korea. Korean J Ophthalmol 2014;28:451-9.

10. Minihan M, Tanner V, Williamson TH. Primary rhegmatogenous retinal detachment: 20 years of change. $\mathrm{Br} J$ Ophthalmol 2001;85:546-8.

11. Tornambe PE. Pneumatic retinopexy: the evolution of case selection and surgical technique. A twelve-year study of 302 eyes. Trans Am Ophthalmol Soc 1997;95:551-78.

12. Ahmad BU, Shah G, Blinder K. Trends \& approaches to repairing detachment. [Internet]. Rev Ophthalmol; 2013 [cited 2013 Apr 5]. Available from: https://www.reviewofophthalmology.com/ article/trends-approaches-to-repairing-detachment.

13. Chan CK, Lin SG, Nuthi AS, Salib DM. Pneumatic retinopexy for the repair of retinal detachments: a comprehensive review (1986-2007). Surv Ophthalmol 2008;53:443-78.

14. Fabian ID, Kinori $M$, Efrati $M$, et al. Pneumatic retinopexy for the repair of primary rhegmatogenous retinal detachment: a 10year retrospective analysis. JAMA Ophthalmol 2013;131:166-71.

15. Ellakwa AF. Long term results of pneumatic retinopexy. Clin Ophthalmol 2012;6:55-9.

16. Martínez-Mujica MT, Retamal J, González R. Scleral buckle versus pneumatic retinopexy for rhegmatogenous retinal detachments. Medwave 2018;18:e7278.

17. Tanaka AC, Goldberg RA. Pneumatic without pexy: a case report of a retinal detachment repair. Retin Cases Brief Rep 2019 Nov 28.; doi: 10.1097/ICB.0000000000000954. [Epub ahead of print]

18. Suzuki AC, Zacharias LC, Tanaka T, et al Case report: pneumatic retinopexy for the treatment of progressive retinal detachment in senile retinoschisis. Arq Bras Oftalmol 2015;78:50-2.

19. Giansanti F, Giuntoli M, Mazzini C, et al. Pneumatic retinopexy for retinal detachment associated with choroidal coloboma. Eur J Ophthalmol 2012;22:680-2. 